\title{
Non-adiabatic Josephson Dynamics in Junctions with in-Gap Quasiparticles
}

\author{
J. Michelsen* and V.S. Shumeiko \\ Department of Microtechnology and Nanoscience, MC2 \\ Chalmers University of Technology, \\ SE-41296 Gothenburg, Sweden
}

(Dated: April 25, 2022)

\begin{abstract}
Conventional models of Josephson junction dynamics rely on the absence of low energy quasiparticle states due to a large superconducting gap. With this assumption the quasiparticle degrees of freedom become "frozen out" and the phase difference becomes the only free variable, acting as a fictitious particle in a local in time Josephson potential related to the adiabatic and non-dissipative supercurrent across the junction. In this article we develop a general framework to incorporate the effects of low energy quasiparticles interacting non-adiabatically with the phase degree of freedom. Such quasiparticle states exist generically in constriction type junctions with high transparency channels or resonant states, as well as in junctions of unconventional superconductors. Furthermore, recent experiments have revealed the existence of spurious low energy in-gap states in tunnel junctions of conventional superconductors - a system for which the adiabatic assumption typically is assumed to hold. We show that the resonant interaction with such low energy states rather than the Josephson potential defines nonlinear Josephson dynamics at small amplitudes.

PACS numbers: $74.50+\mathrm{r}, 74.78 . \mathrm{Na}, 72.10 . \mathrm{Bg}$
\end{abstract}

\section{INTRODUCTION}

During the last twenty years the microscopic theory of the Josephson effect has been undergoing steady development following the advent of novel mesoscopic Josephson structures such as transparent metallic and semiconducting junctions [1], quantum point contacts [2], quantum dot contacts [3], junctions with spin-active interfaces [4]. Much of the theory development for these structures were based on pioneering work by I.O. Kulik [5] 8 . Also important breakthrough was experimental demonstration [9 11] of macroscopic quantum coherence 12] in Josephson junctions, and realization of quantum Josephson circuits (qubits) [13 16].

Functioning of quantum Josephson circuits is based on a fundamental property of Josephson tunnel junctions: nonlinear non-dissipative phase dynamics. Equivalence of Josephson junctions to ideal nonlinear oscillators, pointed out already by Josephson [17], is used in numerous applications in microwave electronics 18. The possibility to quantize the motion of Josephson oscillator [19], and to observe the macroscopic quantum dynamics is essentially based on this fundamental property.

Equation of motion for the superconducting phase difference across the junction stems from Kirchhoff's rule that combines the Josephson tunneling current, $I_{J}(\varphi)=$ $I_{C} \sin \varphi$, and the displacement current through junction capacitor, $(C / 2 e) \ddot{\varphi}$,

$$
\frac{C}{2 e} \ddot{\varphi}+I_{C} \sin \varphi=I_{e}(\varphi, t),
$$

\footnotetext{
*Electronic address: jens.michelsen@chalmers.se
}

where $I_{e}(\varphi, t)$ is a biasing current defined by external circuit, $\hbar=1$. A key assumption behind this equation is a quasi-static form of the Josephson current that extends the static current-phase relation to the non-stationary case of temporal variation of the phase. A justification for this assumption is provided by a wide isotropic superconducting energy gap $\Delta$ that prevents excitation of quasiparticles by temporal variation of the phase at low temperature and small frequency of Josephson plasma oscillation, $k T, \hbar \omega_{p} \ll \Delta$. Thus electrons in the junction remain in equilibrium, and the adiabatic form of the Josephson current is maintained.

Such an approach is relevant for tunnel junctions, but it is not always correct. Notable exceptions are transparent point contacts [20] and resonant quantum dot contacts 21 containing Andreev bound states deep inside the energy gap. Other important exceptions are junctions of d-wave superconductors containing zero energy Andreev surface states [22] and low energy nodal quasiparticles [23]. In such junctions the low energy quasiparticles are involved in the macroscopic dynamics: they are excited and driven away from equilibrium by temporal variation of the phase resulting in significant modification of the Josephson current. How is Eq. (1) then modified in the presence of low energy quasiparticle states?

In this article we suggest an extension of Eq. (1) to describe the non-adiabatic Josephson dynamics in the presence of interaction with quasiparticles. A general equation derived in the next sections has the form,

$$
\begin{array}{r}
\frac{C}{2 e} \ddot{\varphi}+\operatorname{Tr}\left(\hat{I}_{J} \hat{f}\right)=I_{e}, \\
i \dot{\hat{f}}=[\hat{H}, \hat{f}] .
\end{array}
$$

Here the adiabatic Josephson current is replaced by a statistical average of a Josephson current operator, $\hat{I}_{J}$; 
the non-equilibrium quasiparticle density matrix $\hat{f}$ satisfies the Liouville equation with an effective Hamiltonian, $\hat{H}$. The only approximation made during the derivation is a semiclassical approximation for the phase dynamics, otherwise this is an exact equation. As we will show, both the current operator and effective Hamiltonian are expressed through the quasiparticle energy spectrum of the junction and interlevel transition matrix elements.

Eq. (2) has a generic form of equation of motion of a macroscopic particle interacting with a fermionic bath. Usually such problems are treated assuming an equilibrium bath. Here we will consider a non-equilibrium bath consisting of low energy bound Andreev states strongly driven by the phase dynamics. Our main conclusion is that the Rabi dynamics of the Andreev states dramatically modifies the nonlinear properties of macroscopic Josephson dynamics. The physics here resembles well known in nonlinear optics picture of interaction of electromagnetic mode with medium of two-level atoms [24].

The structure of the paper is as follows. In section II we discuss a general approach based on the path integral technique, which is used in Section III to derive Eq. (2). In the next section we discuss the adiabatic limit and establish connection between our method and earlier results for tunnel junctions. Section V is devoted to nonadiabatic effects; we study both the linear and nonlinear quasiparticle response, the main result here is the evaluation of a nonlinear effect of driven low energy Andreev bound states. In Section VI we present the derivation of stochastic Langevin equation generalizing the deterministic Eq. 22.

\section{FORMULATION OF THE PROBLEM}

Consider a general setup of a junction with superconducting electrodes occupying left $(x<0)$ and right $(x>0)$ halfspaces, with an interface at $x=0$ carrying $N$ conducting modes. We will not specify the properties of the interface but rather characterize it, within the quasiclassical approximation, with some electronic transfer matrix. In the following we also adopt common assumptions: (i) the superconductors are described with a BCS mean field theory, (ii) superconducting electrodes maintain local equilibrium implying absence of spatial and temporal variation of the module and phase of the order parameter, $\Delta=$ const, $\chi(\mathbf{r}, t)=\operatorname{sign}(x) \varphi(t) / 2$.

To accurately describe the nonequilibrium dynamics we adopt the path integral approach, introduced by Ambegaokar et al. 25], and adapted for non-equilibrium systems 26-28]. Following this approach we represent the trace of the time dependent statistical operator of the junction $\hat{\rho}(t)=\hat{U}\left(t, t_{0}\right) \hat{\rho}_{0} \hat{U}\left(t_{0}, t\right)$ with the path integral,

$$
Z=\operatorname{Tr}[\hat{\rho}(t)]=\int \mathcal{D} \varphi \mathcal{D} \bar{\psi} \mathcal{D} \psi e^{i S[\varphi, \bar{\psi}, \psi]}
$$

where the action is,

$$
S[\varphi, \bar{\psi}, \psi]=\int_{\mathcal{C}} d t\left(\frac{C}{8 e^{2}} \dot{\varphi}^{2}-U_{e}(\varphi)+\left(\bar{\psi}, \mathcal{G}^{-1} \psi\right)\right) .
$$

The first term in this equation originates from the electrostatic interaction between electrodes and is described within the capacitance approximation 25]; the second term is an inductive energy of the external circuit, and the last term represents the contribution of superconducting electrons. Time integration goes along the forward-backward time contour, $\mathcal{C}=\mathcal{C}_{+}+\mathcal{C}_{-}$. The fermionic fields in the electronic term are written in the Nambu pseudo-spinor representation, $\psi=\left(\psi_{\uparrow}, \bar{\psi}_{\downarrow}\right)^{T}$, and

$$
\mathcal{G}^{-1}=i \partial_{t}-\mathcal{H}(\varphi)-\frac{\dot{\chi}}{2} \sigma_{z}
$$

where

$$
\mathcal{H}(\varphi)=\left(\frac{\boldsymbol{p}^{2}}{2 m}-E_{F}+V(\mathbf{r})\right) \sigma_{z}+\Delta e^{i \sigma_{z} \chi} \sigma_{x},
$$

is the junction Hamiltonian. Here $V(\mathbf{r})$ is the potential defining the interface; superconducting order parameter, $\Delta$, is a scalar in s-wave superconductors, but becomes a nonlocal operator in the case of unconventional d-wave pairing. The last term in Eq. (5) represents the electrical potential needed to preserve electro-neutrality within the electrodes [29],

By virtue of the quadratic form of the Fermionic part of the action (4) one can formally perform the gaussian path integral over the fermionic fields, and reduce the integral to one over the phase degree of freedom 30 33,

$$
Z=\int \mathcal{D} \varphi e^{i S_{0}[\varphi]+\operatorname{Sp} \ln \left(-i \mathcal{G}^{-1}\right)},
$$

here $S_{0}$ comprises the first two terms in Eq. (4), and $\mathrm{Sp}$ denotes the trace over both the quasiparticle states as well as the forward-backward time contour. This transformation in itself, however, does not solve the problem: the obtained effective action contains the contour ordered fermionic Green's function, which needs to be computed by solving the equation of motion. This can only be done under some approximations. The most studied in literature case concerns tunnel junctions where the Green's function is calculated perturbatively using small transparency of the junction, $D \ll 1$ [25, 30, 31]. This is commonly done within the formalism of tunnel Hamiltonian model. This method can be improved and made suitable for transparent junctions, $D \sim 1$, by performing summation of the whole perturbative series 34. However, the tunnel model method does not straightforwardly apply to superconductors with surface states, such as dwave superconductors, since it is based on expansion over bulk Green's functions. The tunnel model must then be modified by considering semi-infinite leads with hard-wall boundaries rather than homogenous leads [35]. An alternative way to calculate the effective action for transparent junctions was suggested in Refs. 29, 36, by using 
exact boundary conditions and an adiabatic approximation for low energy Andreev states. Zaikin and Panuykov [32, 33] suggested a general method for calculating the effective action by establishing a formal relation between the action and the current across the junction. This method, however, requires knowledge of the ac current response to an arbitrary time dependent realization of $\varphi(t)$, which in general is not possible to obtain.

In this paper we suggest an alternative method of calculation of the effective action (7), which is exact in the limit of semiclassical phase dynamics, and universal regarding interaction with any kind of quasiparticle states.

\section{A. Instantaneous Basis}

The central idea of the method is to expand the Nambu fields over an instantaneous eigenbasis of the Hamiltonian (6),

$$
\psi(\mathbf{r}, t)=\sum_{i} \phi_{i}(\mathbf{r} ; \varphi) a_{i}(t)
$$

This allows us to separate the spatial problem from the temporal one by solving the time independent Bogoliubov-de Gennes equation for a fixed value of the phase. Apart from the technical simplifications this basis provides an intuitive understanding of the microscopic processes involved in the Josephson dynamics in terms of transitions between quasiparticle states.

In this basis the action (4) becomes,

$$
S\left[\varphi,\left\{\bar{a}_{i}\right\},\left\{a_{i}\right\}\right]=\int_{\mathcal{C}} d t\left(\frac{C}{8 e^{2}} \dot{\varphi}^{2}-U_{e}(\varphi)+\sum_{i j} \bar{a}_{i} G_{i j}^{-1} a_{j}\right),
$$

where

$$
G_{i j}^{-1}=\left(i \partial_{t}-H_{i j}\right)
$$

represents the quasiparticle Green function in the instantaneous basis, and the Hamiltonian is given by equation,

$$
H_{i j}(\varphi, \dot{\varphi})=E_{i}(\varphi) \delta_{i j}-\dot{\varphi} \mathcal{A}_{i j}
$$

The diagonal elements here are given by the instantaneous eigen energies of the Hamiltonian (6),

$$
\mathcal{H} \phi_{i}=E_{i} \phi_{i}
$$

and the off-diagonal elements are proportional to the matrix elements,

$$
\mathcal{A}_{i j}=\left(\phi_{i}, i \partial_{\varphi} \phi_{j}\right)-\frac{1}{4}\left(\phi_{i}, \operatorname{sign}(x) \sigma_{z} \phi_{j}\right),
$$

of the transitions between the instantaneous eigenstates due to temporal variations of the phase.

The physical meaning of the transition matrix elements can be understood by establishing their connection to the Josephson current operator. Consider a general quantum mechanical equation for the charge current density matrix,

$$
\mathbf{j}_{i j}(\mathbf{r})=\left.\frac{i e}{2 m}\left(\nabla-\nabla^{\prime}\right) \phi_{i}^{\dagger}(\mathbf{r}) \phi_{j}\left(\mathbf{r}^{\prime}\right)\right|_{\mathbf{r}=\mathbf{r}^{\prime}} .
$$

The current through the interface, $S$, is given by equation,

$$
I_{i j}=\int_{S} d \mathbf{n} \cdot \mathbf{j}_{i j}(\mathbf{r})
$$

This is the matrix of the Josephson current operator. If we connect the electrodes in a loop at infinity, we can use the fact that no current is flowing through any other part of the surface of the superconductor so we may extend the surface, $S$, around the whole superconductor and use Gauss law:

$$
2 I_{i j}=\int_{L} d^{3} r \nabla \cdot \mathbf{j}_{i j}(\mathbf{r})-\int_{R} d^{3} r \nabla \cdot \mathbf{j}_{i j}(\mathbf{r}) .
$$

From the explicit form of the Hamiltonian (6) we derive the relation,

$$
\begin{aligned}
(-i \nabla) \cdot \mathbf{j}_{i j} & =-\frac{e}{2 m}\left[\left[-\nabla^{2} \phi_{i}\right]^{\dagger} \phi_{j}-\phi_{i}^{\dagger}\left[-\nabla^{2} \phi_{j}\right]\right] \\
& =-e\left[\left(E_{i}-E_{j}\right) \phi_{i}^{\dagger} \sigma_{z} \phi_{j}+\phi_{i}^{\dagger}\left[\mathcal{H}, \sigma_{z}\right] \phi_{j}\right] .
\end{aligned}
$$

The last term in this equation can be rewritten as

$$
\left[\sigma_{z}, \mathcal{H}\right]=4 i \operatorname{sign}(x) \partial_{\varphi} \mathcal{H}
$$

The current operator then becomes

$$
\begin{aligned}
& I_{i j}= \\
& =-2 i e\left[\left(\phi_{i}, i \partial_{\varphi} \mathcal{H} \phi_{j}-\frac{1}{4}\left(E_{j}-E_{i}\right)\left(\phi_{i}, \operatorname{sign}(x) \sigma_{z} \phi_{j}\right)\right] .\right.
\end{aligned}
$$

By differentiating the eigenvalue equation, $\mathcal{H} \phi_{i}=E_{i} \phi_{i}$, with respect to $\varphi$ one obtains the following identities,

$$
\begin{aligned}
\left(\phi_{i}, i \partial_{\varphi} \mathcal{H} \phi_{i}\right) & =i \partial_{\varphi} E_{i}, \\
\left(\phi_{i}, i \partial_{\varphi} \mathcal{H} \phi_{j}\right) & =\left(E_{j}-E_{i}\right)\left(\phi_{i}, i \partial_{\varphi} \phi_{j}\right), \quad i \neq j .
\end{aligned}
$$

From these one sees that the current matrix elements are given by equations,

$$
\begin{aligned}
I_{i i} & =2 e \partial_{\varphi} E_{i} \\
I_{i j} & =2 e i\left(E_{i}-E_{j}\right) \mathcal{A}_{i j}
\end{aligned}
$$

or

$$
I_{i j}=2 e\left(\frac{\partial E_{i}}{\partial \varphi} \delta_{i j}+i[E, \mathcal{A}]_{i j}\right) .
$$

Thus we conclude that the matrix elements $\mathcal{A}_{i j}$ are related to the off-diagonal matrix elements of the Josephson current operator. 
Towards the end of this section we present a many body Hamiltonian of the junction in the instantaneous eigen basis. To this end we define the conjugate momentum $n$ corresponding to $\varphi$,

$$
n=\frac{\partial L}{\partial \dot{\varphi}}=\frac{C}{4 e^{2}} \dot{\varphi}+\sum_{i j} \mathcal{A}_{i j} \bar{a}_{i} a_{j}
$$

and perform a Legendre transformation of the Lagrangian in Eq. (9), then we promote the variables, $\bar{a}_{i}, a_{i}$, and $\varphi, n$ to operators by imposing standard (anti-) commutation relations to get,

$$
\begin{aligned}
\mathcal{H}_{q} & =\frac{(2 e)^{2}}{2 C}\left(\hat{n}+\sum_{i j} \mathcal{A}_{i j}(\varphi) \hat{a}_{i}^{\dagger} \hat{a}_{j}\right)^{2} \\
& +U_{e}(\varphi)+\sum_{i} E_{i}(\varphi) \hat{a}_{i}^{\dagger} \hat{a}_{i} .
\end{aligned}
$$

\section{EQUATION OF MOTION}

Now we perform integration over the fermionic variables using the instantaneous eigen basis,

$$
Z=\int \mathcal{D} \varphi e^{i S_{0}[\varphi]+\operatorname{Sp} \ln \left(-i G^{-1}\right)}=\int \mathcal{D} \varphi e^{i S_{\mathrm{eff}}[\varphi]}
$$

Defining in a standard manner four Green's function components, depending on wether the time arguments are defined on the forward $(a=+)$ or backward $(a=-)$ part of the contour,

$$
G\left(t, t^{\prime}\right)=G^{a b}\left(t, t^{\prime}\right), \quad t \in \mathcal{C}^{a}, t^{\prime} \in \mathcal{C}^{b},
$$

we write Eq. 10 on the form,

$$
a\left(i \partial_{t}-\hat{H}\left(\varphi^{a}, \dot{\varphi}^{a}\right)\right) \hat{G}^{a b}\left(t, t^{\prime}\right)=\delta^{a b} \delta\left(t-t^{\prime}\right)
$$

Introducing a single particle density matrix through the relation,

$$
\hat{f}(t)=\frac{1}{2 i} \sum_{a} \hat{G}^{a a}(t, t),
$$

we get from Eq. 26 the Liouville equation,

$$
i \dot{\hat{f}}=[\hat{H}, \hat{f}], \quad \hat{H}=\hat{E}-\dot{\varphi} \hat{\mathcal{A}} \text {. }
$$

A semiclassical dynamical equation for the superconducting phase is given by the least action principle formulated in terms of the Wigner variables, $\varphi^{a}=\varphi+a \chi / 2$, and has the form 26 ,

$$
\left.\frac{\delta S_{\mathrm{eff}}[\varphi, \chi]}{\delta \chi}\right|_{\chi=0}=0
$$

To calculate the functional derivative of the fermionic part, we perform a rotation to a single particle basis, in which the dependence on the time derivative of the phase is eliminated from the Hamiltonian. This is achieved by using a unitary matrix $\hat{U}(\varphi)$ satisfying the equation $i \partial_{\varphi} \hat{U}=-\hat{\mathcal{A}} \hat{U}$. Computing the derivative and rotating back to the original basis we find,

$$
\left.\frac{\delta \operatorname{Sp} \ln \left[-i \check{G}^{-1}\right]}{\delta \chi}\right|_{\chi=0}=\frac{i}{2 e} \operatorname{Tr}\left(\hat{I}_{J}(\varphi) \hat{f}\right) \equiv \frac{i}{2 e}\left\langle\hat{I}_{J}\right\rangle
$$

where $\hat{I}_{J}(\varphi)$ is the Josephson current operator defined in Eq. 21. Then introducing external current, $I_{e}=$ $-2 e \partial_{\varphi} U_{e}$, we write equation of motion on the form,

$$
\frac{C}{2 e} \ddot{\varphi}+\operatorname{Tr}\left(\hat{I}_{J} \hat{f}\right)=I_{e}, \quad \hat{I}_{J}=2 e\left(\partial_{\varphi} \hat{E}+i[\hat{E}, \hat{\mathcal{A}}]\right) .
$$

Eqs. 28 and (31) together constitute a central technical result of this paper.

\section{ADIABATIC LIMIT}

In general, in order to solve the coupled equations for the phase (31) and the density matrix 28), one needs to calculate the static quasiparticle energy spectrum, and matrix elements of the interlevel transitions. This is a rather difficult task since the latter quantities are complicated functions of the phase. However, if the quasiparticle spectrum has a gap, and the frequency of the plasma oscillation is small compared to this gap, in other words, if the quasiparticle dynamics is fast on the time scale of the phase variation, one can apply an adiabatic approximation to find the solution.

A formal condition for the adiabatic expansion is $\dot{\varphi} \mathcal{A}_{i j} \ll E_{i}-E_{j}$. In the main approximation, the Hamiltonian in Eq. 28 reads, $\hat{H}=\hat{E}$, and the initial equilibrium density matrix, $\hat{f}(0)=f^{0}(\hat{E}(0))$ defines the solution that remains constant during the phase evolution, $\hat{f}(t)=\hat{f}^{0}$. This implies that the trace in Eq. 31 will only contain the diagonal part of the current operator, and the Josephson current reduces to the adiabatic form,

$$
\begin{array}{r}
I_{J}^{\mathrm{ad}}(\varphi)=2 e \operatorname{Tr}\left(\partial_{\varphi} \hat{E} \hat{f}^{0}\right)=2 e \partial_{\varphi} U_{J} \\
U_{J}(\varphi)=\operatorname{Tr}\left(\hat{E}(\varphi) \hat{f}^{0}\right)
\end{array}
$$

This equation provides a generalization of the tunnel junction equation (1) to the junctions with non-sinusoidal current-phase dependence.

To find the first non-adiabatic correction, it is convenient to expand electronic part in effective action, Eq. (24),

$$
\begin{aligned}
\operatorname{Sp} \ln \left(-i G^{-1}\right) & =\operatorname{Sp} \ln \left(-i\left(G^{\mathrm{ad}}\right)^{-1}\right) \\
& -\sum_{n} \frac{1}{n} \operatorname{Sp}\left(-\check{G}^{\mathrm{ad}} \dot{\varphi} \check{\mathcal{A}}\right)^{n},
\end{aligned}
$$


where $\left(G^{\text {ad }}\right)^{-1}=\delta_{i j}\left(i \partial_{t}-E_{i}(\varphi)\right)$. The first, adiabatic term is given by equation,

$$
\operatorname{Sp} \ln \left(-i\left[G^{\mathrm{ad}}\right]^{-1}\right)=-i \int_{\mathcal{C}} d t U_{J}(\varphi),
$$

consistent with Eq. (32). To see this, we formally introduce $G_{\lambda}^{\text {ad }}=G^{\text {ad }}(\lambda \varphi)$, and rewrite the adiabatic term as 32],

$$
\begin{aligned}
& \operatorname{Sp} \ln \left(-i\left[G^{\mathrm{ad}}\right]^{-1}\right)=\int^{1} d \lambda \frac{d}{d \lambda} \operatorname{Sp} \ln \left(-i\left(G_{\lambda}^{\mathrm{ad}}\right)^{-1}\right) \\
& =-\int_{\mathcal{C}} d t \int^{1} d \lambda \frac{\partial E_{i}(\lambda \varphi)}{\partial \lambda}\left[G_{\lambda}^{\mathrm{ad}}\right]_{i i}(t, t) .
\end{aligned}
$$

Since $\left[G_{\lambda}^{\text {add }}\right]_{i i}(t, t)=i f_{i}^{0}$ does not change with time by virtue of the earlier presented argument, we find,

$$
\begin{aligned}
& \operatorname{Sp} \ln \left(-i\left[G^{\mathrm{ad}}\right]^{-1}\right) \\
& =-i \int_{\mathcal{C}} d t \int^{\varphi} d \varphi^{\prime} \operatorname{Tr}\left(\frac{\partial \hat{E}\left(\varphi^{\prime}\right)}{\partial \varphi^{\prime}} \hat{f}^{0}\right)=-i \int_{\mathcal{C}} d t U_{J} .
\end{aligned}
$$

The first order non-adiabatic term in the series, Eq. 33, cancels since $\hat{G}^{\text {ad }}$ is diagonal while $\hat{\mathcal{A}}$ is purely offdiagonal, which implies that the trace of their product is zero. Keeping then only the second order correction we find,

$$
\begin{aligned}
& \operatorname{Sp} \ln \left(-i \check{G}^{-1}\right)=-i \int_{\mathcal{C}} d t U_{J}(\varphi) \\
& -\frac{1}{2} \int_{\mathcal{C}} d t \int_{\mathcal{C}} d t^{\prime} \dot{\varphi} \mathcal{A}_{i j}(\varphi) G_{j j}^{\mathrm{ad}}\left(t, t^{\prime}\right) \dot{\varphi}^{\prime} \mathcal{A}_{j i}\left(\varphi^{\prime}\right) G_{i i}^{\mathrm{ad}}\left(t^{\prime}, t\right) .
\end{aligned}
$$

When the occupied and unoccupied states are separated by a large gap, the product

$$
G_{j j}^{\mathrm{ad}}\left(t, t^{\prime}\right) G_{i i}^{\mathrm{ad}}\left(t^{\prime}, t\right) \sim e^{-i \int_{t}^{t^{\prime}} d t^{\prime \prime} \varepsilon_{i j}\left(\varphi^{\prime \prime}\right)}, \quad \varepsilon_{i j}=E_{i}-E_{j},
$$

oscillates rapidly on the scale of variations of the phase, and we can treat this object in the local approximation. This gives us,

$$
\operatorname{Sp} \ln \left(-i \check{G}^{-1}\right)=-i \int_{\mathcal{C}} d t\left[U_{J}(\varphi)+\frac{\delta C(\varphi)}{8 e^{2}} \dot{\varphi}^{2}\right],
$$

where

$$
\delta C(\varphi)=2 e^{2} \sum_{i j} \frac{\left|\mathcal{A}_{i j}(\varphi)\right|^{2} f_{i}\left(1-f_{j}\right)}{\varepsilon_{i j}(\varphi)}
$$

represents a phase dependent correction to the junction capacitance.
Let us explicitly evaluate the contribution to Eq. 40. of the Andreev bound states in a tunnel junction. In tunnel junctions, Andreev energy levels are located very close to the gap edges [37] having the level spacing, $\varepsilon=2 \Delta \sqrt{1-D \sin ^{2} \frac{\varphi}{2}} \approx 2 \Delta$. The transitions connect only Andreev states of the same conducting mode with transition matrix elements [38,

$$
\mathcal{A}=i \sqrt{R D} \Delta \frac{|\sin \varphi / 2|}{2 \varepsilon} \approx \frac{i \sqrt{D}}{4} \sin \frac{\varphi}{2} .
$$

Computing the correction to the capacitance using these expressions we find the phase dependent correction in the zero temperature limit to be, $\delta C_{\varphi} \approx\left(D e^{2} / 32 \Delta\right) \cos \varphi$, per conducting mode. This is consistent with the result of the tunnel model calculation in Refs. [25, 30.

\section{NON-ADIABATIC DYNAMICS}

\section{A. Linear response}

The non-adiabatic dynamics essentially results from the resonant response of low energy quasiparticles to the phase variation. In this section we consider the linear quasiparticle response and compute the non-adiabatic correction to the frequency of Josephson plasma oscillation.

Consider small deviations from an equilibrium configuration, $\varphi=\varphi_{0}$ and $\hat{f}=\hat{f}^{0}$ determined by the equation, $I_{J}^{\mathrm{ad}}\left(\varphi_{0}\right)=\operatorname{Tr}\left(\hat{I}\left(\varphi_{0}\right) \hat{f}^{0}\right)=I_{e}\left(\varphi_{0}\right)$. Straightforward linearization of Eqs. (28) and (31) with respect to small deviations of the phase, $\varphi(t)-\varphi_{0}$, and the density matrix, $\hat{f}(t)-\hat{f}^{0}$, leads to the dispersion equation for the plasma oscillation,

$$
\left(-\omega^{2}+\omega_{p}^{2}+\omega \gamma_{0}(\omega)\right) \varphi_{\omega}=0,
$$

where

$$
\omega_{p}^{2}=\frac{2 e}{C} \frac{\partial I_{J}^{\mathrm{ad}}\left(\varphi_{0}\right)}{\partial \varphi}
$$

is the adiabatic plasma frequency, and $\gamma_{0}(\omega)$ denotes the linear response of the quasiparticles,

$$
\gamma_{0}(\omega)=\frac{4 e^{2}}{C} \sum_{i j} \frac{\varepsilon_{i j}\left|\mathcal{A}_{i j}\right|^{2}\left(f_{i}^{0}-f_{j}^{0}\right)}{\varepsilon_{i j}-(\omega+i 0)} .
$$

The linear response of quasiparticle is a relevant approximation at small phase oscillation when the quasiparticles have a continuous energy spectrum and the transferred energy is dispersed across a large phase space volume resulting in weak non-equilibrium. As such the dispersion equation (42) can be applied, for example, to the low energy itinerant states in the nodal regions of high-Tc superconductors, or to broadened Andreev bound states in disordered junctions. However, the linear approximation does not apply to spectroscopically narrow Andreev bound states, whose response is essentially nonlinear even at small phase amplitude. 


\section{B. Resonant interaction with Andreev levels}

Now we consider the nonlinear dynamics of the phase driven by small oscillating current $I_{e}(t)=I_{e} \cos \omega t$, at a frequency not far from the resonant frequency, $\delta=$ $\omega-\omega_{p} \ll 1$, in the presence of resonant interaction with weakly broadened low energy Andreev levels. Such levels may exist in transparent electronic conducting modes close to $\varphi_{0}=\pi$, in electronic modes with resonant transmissivity, or in surface modes of d-wave superconductors. The exact nature of these states does not play any role for our analysis. The important properties are: (i) the phase variations do not change the electronic momentum hence do not induce quasiparticle transitions among the conducting modes, (ii) therefore transitions only occur between pairs of Andreev states within the same conducting mode, (iii) the Andreev levels are well separated from the continuum states of the mode. Under these assumptions, the Hamiltonian in Eq. 28 truncated to the Andreev level subspace consists of a sum of independent two-level Hamiltonians, and the density matrix factorizes to the product of two-level density matrices parameterized with the conduction mode number, $\hat{f}(n)$. The non-adiabatic current then becomes:

$$
\begin{aligned}
& \left\langle I_{J}\right\rangle-I_{J}^{\mathrm{ad}}(\varphi)= \\
& 2 e \sum_{n}\left(\frac{\partial \varepsilon}{\partial \varphi}\left(f_{z}-f_{z}^{0}\right)-\varepsilon\left(\mathcal{A} f_{-}+\mathcal{A}^{*} f_{+}\right)\right),
\end{aligned}
$$

where, $\varepsilon=E_{1}-E_{2}$, is the level spacing between two Andreev states associated with a specific mode $n$ and, $i \mathcal{A}=\mathcal{A}_{12}$, is the corresponding transition matrix element (we skip index $n$ for brevity). Similarly $f_{z}=f_{11}-f_{22}$ and $f_{+}=f_{12}=\left(f_{-}\right)^{*}$, are the corresponding elements of the two-level density matrix satisfying the Bloch-Redfield equation,

$$
\begin{aligned}
\dot{f}_{+} & =\left(-i \varepsilon-\Gamma_{2}\right) f_{+}+2 \dot{\varphi} \mathcal{A} f_{z} \\
\dot{f}_{z} & =-\dot{\varphi} \mathcal{A} f_{+}^{*}-\dot{\varphi} \mathcal{A}^{*} f_{+}-\Gamma_{1}\left(f_{z}-f_{z, 0}\right),
\end{aligned}
$$

where we have added phenomenological decay rates $\Gamma_{1}$ and $\Gamma_{2}$ originating, e.g., from some weak inelastic interaction with the continuum states.

To separate the fast and slow resonant dynamics, we parameterize the phase as,

$$
\begin{aligned}
\varphi(t) & =\frac{1}{2}\left(\varphi_{\omega}(t) e^{-i \omega t}+\text { c.c. }\right) \\
\dot{\varphi}(t) & =\frac{\omega}{2 i}\left(\varphi_{\omega}(t) e^{-i \omega t}-\text { c.c. }\right),
\end{aligned}
$$

where the complex variable, $\varphi_{\omega}(t)=r(t) e^{i \vartheta(t)}$, depends on the amplitude of oscillations, $r(t)$, and the time dependent phase shift, $\vartheta(t)$. Using a similar separation for the fast and slow parts of the off-diagonal elements of the density matrix,

$$
f_{+}(t)=f_{\omega}(t) e^{-i \omega t}
$$

we get, after expanding to first order in, $\varphi-\varphi_{0}$, and averaging over fast variables (note $\mathcal{A}_{0}=\mathcal{A}\left(\varphi_{0}\right)$ and $\varepsilon_{0}=$ $\left.\varepsilon\left(\varphi_{0}\right)\right)$,

$$
\begin{aligned}
& \dot{f}_{\omega}=-i\left(\varepsilon_{0}-\omega-i \Gamma_{2}\right) f_{\omega}-i \omega \mathcal{A}_{0} \varphi_{\omega} f_{z} \\
& \dot{f}_{z}=i \frac{\omega}{2}\left(\mathcal{A}_{0} \varphi_{\omega} f_{\omega}^{*}+\text { c.c. }\right)-\Gamma_{1}\left(f_{z}-f_{z, 0}\right) .
\end{aligned}
$$

The regime relevant for our discussion corresponds to slow variation of the phase oscillation envelope, $\varphi_{\omega}$, on the time scale of the Andreev state relaxation. Then the Andreev state density matrix will adiabatically follow the evolution of the phase amplitude (in the rotating frame), and we restrict ourselves to the quasi-stationary solutions, $\dot{f}_{\omega}, \dot{f}_{z} \approx 0$, to find from the first equation in 49,

$$
f_{\omega}=\frac{\omega \mathcal{A}_{0} \varphi_{\omega}}{\left(\omega+i \Gamma_{2}\right)-\varepsilon_{0}} f_{z}
$$

Inserting this expression into the current we find,

$$
\begin{array}{r}
\left\langle I_{J}\right\rangle=I_{J}^{\mathrm{ad}}(\varphi)+2 e \sum_{n}\left(\frac{\partial \varepsilon_{0}}{\partial \varphi}\left(f_{z}-f_{z}^{0}\right)\right. \\
\left.-\frac{\omega \varepsilon_{0}\left|\mathcal{A}_{0}\right|^{2} f_{z}}{\left(\omega+i \Gamma_{2}\right)-\varepsilon_{0}} \varphi_{\omega} e^{-i \omega t}+\text { c.c. }\right) .
\end{array}
$$

Eq. (51) illustrates the principal effect of the resonant interaction between the phase and the Andreev levels: the phase oscillation drives the Andreev levels to a nonequilibrium state determined by the stationarity condition,

$$
f_{z}=f_{z}^{0}-\frac{\Omega^{2}(r)\left(\Gamma_{2} / \Gamma_{1}\right)}{\left(\varepsilon_{0}-\omega\right)^{2}+\Gamma_{2}^{2}+\Omega^{2}(r)\left(\Gamma_{2} / \Gamma_{1}\right)} f_{z}^{0} .
$$

Here $\Omega(r)=\left|\omega \mathcal{A}_{0} r\right|$ is the amplitude dependent Rabi frequency of the Andreev two-level system associated with specific mode $n$. The first term inside the bracket in Eq. (51) produces a nonlinear modulation of the Josephson potential due the nonequilibrium population of the Andreev levels. The second term causes a nonlinear damping of the phase oscillation, similar to the imaginary part of the linear response, although it now depends on the nonequilibrium population of the Andreev levels.

For the levels close to the resonance, $\varepsilon_{0} \approx \omega$, the diagonal elements are approximately given by

$$
f_{z} \approx \frac{\Gamma^{2}}{\Gamma^{2}+\Omega^{2}(r)} f_{z}^{0}
$$

where, $\Gamma=\sqrt{\Gamma_{1} \Gamma_{2}}$. Thus in the limit of $\Omega(r) \ll \Gamma$, i.e. $r \ll \Gamma /\left|\omega \mathcal{A}_{0}\right|$, we recover the linear response regime. In the opposite limit, $\Omega(r) \gg \Gamma$, i.e. $r \gg \Gamma /\left|\omega \mathcal{A}_{0}\right|$, the levels become saturated, $f_{z} \approx 0$, and can no longer absorb energy from the phase oscillation, thus the damping decreases for large amplitude of phase oscillation. 


\section{Nonlinear phase dynamics}

To see how the nonlinear quasiparticle response manifests itself in the junction dynamics we write down the equation of motion for the slowly varying amplitudes, $\varphi_{\omega}$, and introduce a nonlinear response function, $\gamma(r)=\gamma^{\prime}(r)+i \gamma^{\prime \prime}(r)$, defined through the relation,

$$
\frac{2 e}{C}\left(\left\langle I_{J}\right\rangle-I_{J}^{\mathrm{ad}}(\varphi)\right)=\omega \gamma(\omega, r) \varphi_{\omega} e^{-i \omega t}+c . c .
$$

in terms of which the averaged equation for the envelope becomes,

$$
-2 i \omega_{p} \dot{\varphi}_{\omega}+\left[-2 \omega_{p} \delta+\omega_{p} \gamma\left(\omega_{p}, r\right)\right] \varphi_{\omega}=\frac{e}{C} I_{e}
$$

The stationary solutions to this equation, $\dot{\varphi}_{\omega}=0$, connect resonant amplitude and detuning $\delta$,

$$
\delta=\frac{1}{2} \gamma^{\prime}(r) \pm \frac{1}{2 r} \sqrt{\left(e I_{e} / C \omega_{p}\right)^{2}-\left(\gamma^{\prime \prime}(r)\right)^{2} r^{2}} .
$$

The two solutions correspond to the stable/unstable branches of the function $r(\delta)$ as illustrated on Fig. 1. The maximum response, $r_{m}$, is found where the two branches coincide, i.e. $r_{m} \gamma^{\prime \prime}\left(r_{m}\right)=e I_{e} / C \omega_{p}$.

To make a quantitative analysis we write $\sum_{n}=$ $\int d \varepsilon \nu(\varepsilon)$, where $\nu(\varepsilon)=\sum_{n} \delta\left(\varepsilon-\varepsilon_{0}(n)\right)$. If the density of states $\nu(\varepsilon)$ is a smooth function close to the resonance the integration can be explicitly performed, giving,

$$
\begin{aligned}
\gamma^{\prime}(r) & =\gamma_{0}^{\prime}-\frac{\partial_{\varphi}^{2} \bar{\varepsilon}_{0} r^{2}}{\Gamma_{1}} \frac{\Gamma \gamma_{0}^{\prime \prime}}{\sqrt{\bar{\Omega}(r)^{2}+\Gamma^{2}}}, \\
\gamma^{\prime \prime}(r) & =\frac{\Gamma \gamma_{0}^{\prime \prime}}{\sqrt{\bar{\Omega}(r)^{2}+\Gamma^{2}}},
\end{aligned}
$$

where $\gamma_{0}^{\prime \prime}$ is the imaginary part of the linear response (44),

$$
\gamma_{0}^{\prime \prime}=\frac{4 e^{2}}{C} \omega\left|\overline{\mathcal{A}}_{0}\right|^{2} \nu(\omega) f_{z}^{0}(\omega / 2),
$$

and bars indicate the values of the functions at the resonance. With this expression we find the maximum response amplitude,

$$
r_{\mathrm{m}}=\tilde{I}_{e}\left(1-\left(\tilde{I}_{e} / I^{*}\right)^{2}\right)^{-1 / 2},
$$

where

$$
\tilde{I}_{e}=\frac{e I_{e}}{C \omega_{p} \gamma_{0}^{\prime \prime}}=\frac{\omega_{p}}{2 \gamma_{0}^{\prime \prime}} \frac{I_{e}}{I_{C}}
$$

is the dimensionless driving current, and

$$
I^{*}=\frac{\Gamma}{\omega_{p}\left|\overline{\mathcal{A}}_{0}\right|} .
$$

This result shows that the response has an explosive instability manifested by a divergency of the oscillation amplitude when the driving current amplitude reaches the critical value $\tilde{I}_{e}=I^{*}$. We emphasize that this current is much smaller than the Josephson critical current, $I_{C}$, which sets the scale for the nonlinear behavior of the adiabatic junctions. This instability is easy to understand noticing that the damping produced by the Andreev states decreases with amplitude of oscillation, and, on the other hand, it is the damping value that limits the resonance response amplitude. To eliminate the divergency, one has to take into account other damping mechanisms, which are weaker than the linear damping by the Andreev states.

If we turn off the external drive, $I_{e}=0$, we find from Eq. (55) the equation for the decay of the oscillation amplitude, $\dot{r}=-\gamma^{\prime \prime}(r) r / 2$. For $r>\Gamma /\left|\overline{\mathcal{A}}_{0}\right| \omega_{p}$, we find that the plasma oscillation decays linearly with time with the rate, $\dot{r} \approx-\left(\Gamma \gamma_{0}^{\prime \prime} /\left|\overline{\mathcal{A}}_{0}\right| \omega_{p}\right)=$ const, until it enters the linear regime, $r<\Gamma /\left|\overline{\mathcal{A}}_{0}\right| \omega_{p}$, where the decay crosses over to an exponential time dependence, $r \sim \exp \left(-\gamma_{0}^{\prime \prime} t\right)$.

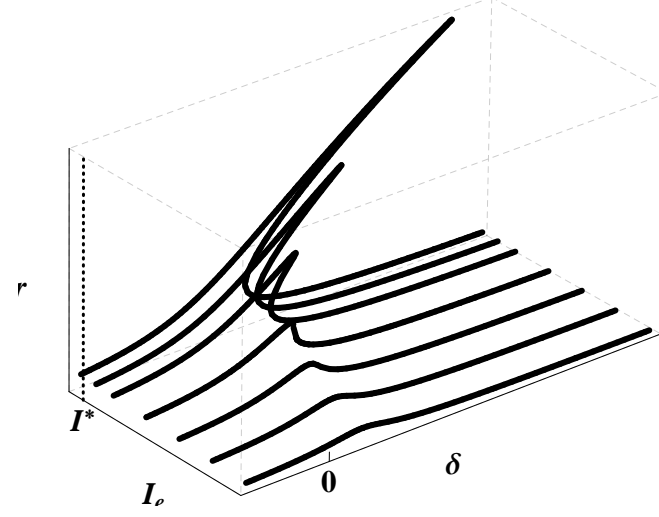

FIG. 1: Effect of resonant interaction with spectroscopically sharp Andreev bound states on non-linear response of the junction. Phase oscillation amplitude as a function of detuning shown for different amplitudes of driving current.

\section{LANGEVIN EQUATION}

The classical equation of motion (31) is deterministic and thus does not include the fluctuations originating from the coupling of the phase to the quasiparticles. In this section we shall outline how these effects can be taken into account. The method we adopt results in a quantum Langevin equation [26, 28, 39, although as we shall show, the stochastic force in this case generally has non-gaussian properties.

Expectation values of any function of phase, $F(\varphi)$, is given by

$$
\langle F\rangle\left(t_{1}\right)=\int d \varphi_{1} F\left(\varphi_{1}\right) \rho_{\text {red }}\left(\varphi_{1}, \varphi_{1}, t_{1}\right),
$$

where $\rho_{\text {red }}\left(\varphi_{1}, \varphi_{1}, t_{1}\right)$ is the reduced density matrix. Noticing that the partition function (3) is given by 
the trace over the reduced density matrix, $Z=$ $\int d \varphi_{1} \rho_{\text {red }}\left(\varphi_{1}, \varphi_{1}, t\right)$, we are able to write the diagonal elements in terms of the Wigner variables $\varphi, \chi$, on the form,

$$
\rho_{\text {red }}\left(\varphi_{1}, \varphi_{1}, t_{1}\right)=\int_{\varphi\left(t_{1}\right)=\varphi_{1}} \mathcal{D} \varphi \int_{\chi\left(t_{1}\right)=0} \mathcal{D} \chi e^{i S[\varphi, \chi]},
$$

where the limits on the functional integrals indicate that the endpoints, $\varphi\left(t_{1}\right), \chi\left(t_{1}\right)$, of the trajectories are to be held fixed.

To zeroth order in the saddle point approximation, $S[\varphi, \chi] \approx \int d t \chi(t)(\delta S[\varphi, 0] / \delta \chi(t))$, only the classical path, $\varphi_{c}(t)$, is realized and the density matrix is written:

$$
\begin{aligned}
& \rho_{\text {red }}\left(\varphi_{1}, \varphi_{1}, t\right) \\
& =\int_{\varphi\left(t_{1}\right)=\varphi_{1}} \mathcal{D} \varphi \int_{\chi\left(t_{1}\right)=0} \mathcal{D} \chi \exp \left(i \int d t \chi(t) \frac{\delta S[\varphi, 0]}{\delta \chi(t)}\right) \\
& =\int_{\varphi\left(t_{1}\right)=\varphi_{1}} \mathcal{D} \varphi \delta\left[\frac{\delta S[\varphi, 0]}{\delta \chi(t)}\right],
\end{aligned}
$$

where, $\delta[\ldots]$, denotes a delta functional. Average quantities are then entirely determined by the classical path $\langle F\rangle\left(t_{1}\right)=F\left(\varphi_{c}\left(t_{1}\right)\right)$.

To go beyond this deterministic description and include fluctuations we can expand the action around the saddle point, $\chi=0$, to second order,

$$
\begin{aligned}
S[\varphi, \chi] & \approx \int d t \chi(t) \frac{\delta S[\varphi, 0]}{\delta \chi(t)} \\
& +\frac{1}{2} \int d t d t^{\prime} \chi(t) \frac{\delta^{2} S[\varphi, 0]}{\delta \chi(t) \delta \chi\left(t^{\prime}\right)} \chi\left(t^{\prime}\right) .
\end{aligned}
$$

Here the kernel,

$$
\begin{aligned}
& i \frac{\delta^{2} S[\varphi, 0]}{\delta \chi(t) \delta \chi\left(t^{\prime}\right)} \\
& =-\frac{i}{(2 e)^{2}} \sum_{a b} \operatorname{Tr}\left[\hat{G}^{a b}\left(t, t^{\prime}\right) \hat{I}\left(t^{\prime}\right) \hat{G}^{b a}\left(t^{\prime}, t\right) \hat{I}(t)\right] \\
& =-\frac{1}{(2 e)^{2}} \mathcal{S}_{I}[\varphi]\left(t, t^{\prime}\right)
\end{aligned}
$$

is given by the symmetrized current-current correlation function, $\mathcal{S}_{I}[\varphi]\left(t, t^{\prime}\right)$, which is a functional of $\varphi$ due to the dependence of $\hat{G}^{a b}$ and $\hat{I}$ on $\varphi(t)$.

We decouple the quadratic term in $\chi$ by introducing an auxiliary variable $I_{\xi}$ which shall later be interpreted as a stochastic current 39,

$$
\begin{aligned}
& \exp \left(-\frac{1}{2} \int d t d t^{\prime} \chi(t) \frac{1}{(2 e)^{2}} \mathcal{S}_{I}[\varphi]\left(t, t^{\prime}\right) \chi\left(t^{\prime}\right)\right) \\
& =\int \mathcal{D} I_{\xi} e^{-\frac{i}{2 e} \int d t I_{\xi}(t) \chi(t)} P\left[\varphi, I_{\xi}\right]
\end{aligned}
$$

where $P\left[\varphi, I_{\xi}\right]$ denotes the functional distribution

$$
P\left[\varphi, I_{\xi}\right]=\mathcal{N}[\varphi] e^{-\frac{1}{2} \int d t d t^{\prime} I_{\xi}(t) \mathcal{S}_{I}^{-1}[\varphi]\left(t, t^{\prime}\right) I_{\xi}\left(t^{\prime}\right)},
$$

where $\mathcal{N}[\varphi]=\left(\operatorname{det} S_{I}^{-1}[\varphi]\right)^{-1 / 2}$. The density matrix can then be written as

$$
\begin{aligned}
& \rho_{\text {red }}\left(\varphi_{1}, \varphi_{1}, t_{1}\right) \\
& =\int \mathcal{D} \xi \int \mathcal{D} \varphi \delta\left[\frac{\delta S[\varphi, 0]}{\delta \chi(t)}-I_{\xi}(t)\right] P\left[\varphi, I_{\xi}\right] .
\end{aligned}
$$

The delta functional selects a single trajectory, $\varphi_{\xi}$, for each realization of, $I_{\xi}$, determined by the classical equation,

$$
\frac{\delta S[\varphi, 0]}{\delta \chi(t)}=I_{\xi}(t) \Rightarrow \frac{C}{2 e} \ddot{\varphi}_{\xi}+\langle I\rangle\left[\varphi_{\xi}\right]=I_{\xi},
$$

where, for the sake of convenience, we assumed an unbiased junction. Eq. (69), is a stochastic equation and averages are given by,

$$
\langle F\rangle\left(t_{1}\right)=\left\langle F\left(\varphi_{\xi}\left(t_{1}\right)\right)\right\rangle_{\xi},
$$

where $\langle\ldots\rangle_{\xi}=\int \mathcal{D} I_{\xi}(\ldots) P\left[\varphi_{\xi}, I_{\xi}\right]$. In contrast to the conventional theory of quantum Langevin equations the functional distribution, $P\left[\varphi_{\xi}, I_{\xi}\right]$, is in general nongaussian due to the dependence of the symmetrized current correlation function on $\varphi_{\xi}=\varphi\left[I_{\xi}\right]$. This is a consequence of non-equilibrium nature of the fermionic bath strongly coupled to the phase variable.

The stochastic force becomes gaussian under the linear response approximation. We consider small deviations from a classical equilibrium configuration, $\varphi(t)=\varphi_{0}$ and $\hat{f}(t)=f^{0}$, and get the equation,

$$
\left(-\omega^{2}+\omega_{p}^{2}+\omega \gamma_{0}(\omega)\right) \delta \varphi(\omega)=\frac{2 e}{C} I_{\xi}(\omega) .
$$

The functional distribution can be taken at the equilibrium value, $P\left[\varphi_{0}, \xi\right]$, which then becomes Gaussian and the stochastic current, $I_{\xi}$, satisfies the typical relations for Gaussian noise:

$$
\left\langle I_{\xi}(t)\right\rangle_{\xi}=0, \quad\left\langle I_{\xi}(t) I_{\xi}\left(t^{\prime}\right)\right\rangle_{\xi}=\mathcal{S}_{I}^{0}\left(t-t^{\prime}\right),
$$

where

$$
\begin{aligned}
& \mathcal{S}_{I}^{0}(\omega)=\operatorname{coth}\left(\frac{\omega}{2 T}\right) \sum_{i j}\left|I_{i j}\right|^{2}\left(f_{i}^{0}-f_{j}^{0}\right) \pi \delta\left(\omega-\varepsilon_{i j}\right) \\
= & (2 e)^{2} \omega^{2} \operatorname{coth}\left(\frac{\omega}{2 T}\right) \sum_{i j}\left|\mathcal{A}_{i j}\right|^{2}\left(f_{i}^{0}-f_{j}^{0}\right) \pi \delta\left(\omega-\varepsilon_{i j}\right) \\
= & C \omega \operatorname{coth}\left(\frac{\omega}{2 T}\right) \operatorname{Im} \gamma_{0}(\omega) .
\end{aligned}
$$

Thus the fluctuating current is related to the dissipative response by the quantum fluctuation dissipation theorem. 


\section{CONCLUSIONS}

We have presented a general theory framework for describing non-adiabatic dynamics of Josephson junctions with low energy quasiparticle states. The theory applies to a wide class of Josephson junctions including transparent mesoscopic contacts based on 2DEG, nanowires, quantum dots, and also junctions of unconventional superconductors. It was shown that in the classical limit the equation of motion for the phase must be solved together with a Liouville equation for density matrix of low energy fermionic states. Furthermore, we illustrated how the dynamics of such systems can differ significantly from the adiabatic (tunnel) junctions, by investigating the resonant dynamics of the phase and low energy Andreev bound states. It was shown that nonlinear, two-state dynamics of the Andreev bound states, rather than an adiabatic Josephson energy, defines the nonlinear macroscopic dynamics of the junction.

This work was supported by the Swedish Research Council (VR), and the European FP7-ICT Project MIDAS.
[1] H. Takayanagi and T. Kawakami, Phys. Rev. Lett. 54, 2449 (1985).

[2] N. van der Post, et al., Phys. Rev. Lett. 732611 (1994).

[3] P. Jarillo-Herrero, J.A. van Dam, and L.P. Kouwenhoven, Nature 439, 953 (2006).

[4] V. V. Ryazanov, et al., Phys. Rev. Lett. 86, 2427 (2001).

[5] I.O. Kulik, Zh. Eksp. Teor. Fiz. 30, 944 (1969) [Sov. Phys. JETP 57, 1745 (1969)].

[6] I.O. Kulik and A.N. Omel'yanchuk, Zh. Eksp. Teor. Fiz. Pis. Red. 21, 216 (1975) [JETP Lett. 21 96].

[7] I.O. Kulik, Zh. Eksp. Teor. Fiz. 49, 1211 (1965) [Sov. Phys. JETP 22, 841 (1966)].

[8] I.O. Kulik and R.I. Shekhter, Zh. Eksp. Teor. Fiz. 68, 623 (1975) [ Sov. Phys. JETP 41, 308 (1975)].

[9] Y. Nakamura, C.D. Chen, and J.S. Tsai, Phys. Rev. Lett. 79, 2328-2331 (1997).

[10] V. Bouchiat, D. Vion, P. Joyez, D. Esteve, and M. H. Devoret, Phys. Scr. T76, 165 (1998).

[11] J. R. Friedman, V. Patel, W. Chen, S. K. Tolpygo. and J. E. Lukens, Nature 406, 43-46 (2000).

[12] A. J. Leggett, and A. J. Garg, Phys. Rev. Lett. 54, 857 (1985).

[13] Y. Makhlin, G. Schön and A. Shnirman Rev. Mod. Phys. 73, 357 (2001)

[14] G. Wendin, and V. S. Shumeiko, Low Temp. Phys. 33, 724 (2007).

[15] J. Clarke, F. K. Wilhelm, Nature 453, 1031 (2008).

[16] R. J. Schoelkopf and S. M. Girvin, Nature 451, 664 (2008).

[17] B. D. Josephson, Rev. Mod. Phys. 36, 216 (1964).

[18] K.K. Likharev, Dynamics of Josephson Junctions and Circuits (Gordon, 1986).

[19] D.J. Scalapino, in Tunneling Phenomena in Solids, ed. E. Burstein and S. Lundqvist (Plenum, 1969).

[20] C. W. J. Beenakker and H. van Houten, Phys. Rev. Lett. 66, 3056 (1991).
[21] G. Wendin and V.S. Shumeiko, Superlatt. and Microstr. 20, 569 (1996).

[22] T. Löfwander, V.S. Shumeiko, and G. Wendin, Supercond. Sci. Technol. 14, R53 (2001).

[23] D.J. Scalapino, Phys. Rep. 250, 329 (1995).

[24] L. Allen and J.H. Eberly, Optical Resonance and TwoLevel Atoms, (Dover, 1987).

[25] V. Ambegaokar, U. Eckern and G. Schön Phys. Rev. Lett. 48, 1745 (1982).

[26] A. Kamenev, arXiv:cond-mat/0412229 bv2, (2005).

[27] H. Kleinert, Path Integrals in Quantum Mechanics, Statistics, Polymer Physics, and Financial Markets (World Scientific, 1999).

[28] U. Weiss, Quantum Dissipative Systems (World Scientific, 2004).

[29] A. Zazunov, V.S. Shumeiko, E.N. Bratus', and G. Wendin, Phys. Rev. B 71, 214505 (2005).

[30] U. Eckern, G. Schön and V. Ambegaokar Phys. Rev. B 30, 6419 (1984).

[31] A. I. Larkin and Yu. N. Ovchinnikov Phys. Rev. B 28, 6281 (1983).

[32] A. D. Zaikin and G. Schön Phys. Rep. 198, 237 (1990).

[33] A. D. Zaikin and S. V. Panyukov Zh. Eksp. Teor. Fiz. 89, 242 (1985) [ Sov. Phys. JETP 62, 137 (1985)]

[34] A. Martin-Rodero, F. J. Garcia-Vidal and A. Levy Yeyati Phys. Rev. Lett. 72, 554 (1994)

[35] J. C. Cuevas and M. Fogelström Phys. Rev. B 64, 104502 (2001).

[36] A. Zazunov, V. S. Shumeiko, E. N. Bratus', J. Lantz, and G. Wendin Phys. Rev. Lett. 90, 0870003 (2003).

[37] A. Furusaki and M. Tsukada, Physica B 165-166, 967 (1990).

[38] V.S. Shumeiko, G. Wendin, and E.N. Bratus', Phys. Rev. B 48, 13129 (1993).

[39] A. Schmid, J. Low Temp. Phys. 49, 609 (1982). 